\title{
Sulfatase 2 facilitates lymphangiogenesis in breast cancer by regulating VEGF-D
}

\author{
CHENFANG ZHU $^{1 *}, \mathrm{XIAOLIANG} \mathrm{QI}^{1 *}, \mathrm{XIN} \mathrm{ZHOU}^{2}, \mathrm{XIN} \mathrm{NIE}^{1}$ and $\mathrm{YAN} \mathrm{GU}^{1}$ \\ ${ }^{1}$ Department of General Surgery, Shanghai Ninth People's Hospital, Affiliated with Shanghai Jiaotong University, \\ School of Medicine, Shanghai 200011, P.R. China; ${ }^{2}$ Department of Surgery, George Washington University, \\ Washington, DC 20052, USA
}

Received May 26, 2016; Accepted July 1, 2016

DOI: $10.3892 /$ or.2016.5143

\begin{abstract}
In our previous studies, sulfatase 2(Sulf2) was found to upregulate vascular endothelial growth factor-D (VEGF-D) expression in breast cancer. As VEGF-D plays an important role in lymphangiogenesis, we hypothesized that Sulf2 facilitates lymphangiogenesis in breast cancer by regulating VEGF-D. To evaluate the functions of Sulf 2 on lymphangiogenesis in breast cancer, proliferation, apoptosis, cell cycle, cell mobility and tube-formation of lymphatic endothelial cells (LECs) were measured in vitro. Lymphangiogenesis in nude mouse ears and breast cancer xenografts were examined in vivo. Furthermore, the expression levels of related signaling pathway genes were screened and verified in LECs. We found that Sulf 2 significantly increased the mobility and tube formation of the LECs, inhibited cisplatin-induced LEC apoptosis, but had no effect on cell proliferation and the cell cycle. Moreover, recombinant Sulf2 (rSulf2) combined with VEGF-D further promoted the proliferation, cell cycle, mobility and tube-like structure formation in the LECs, and at the same time inhibited cisplatin-induced apoptosis especially in the late stage. Sulf2 also significantly increased the density of lymphatic vessels in mouse ears and breast cancer xenografts in vivo. AKT1 was also shown to be upregulated and activated by Sulf2. Our results confirmed that Sulf2 facilitated lymphangiogenesis in breast cancer cells by regulating VEGF-D and that the AKT1-related signaling pathway was involved.
\end{abstract}

\section{Introduction}

Extracellular sulfatases, especially heparan endosulfatases (Sulfs), play important roles in cancer progression by

Correspondence to: Dr Yan Gu, Department of General Surgery, Shanghai Ninth People's Hospital, Affiliated with Shanghai Jiaotong University, School of Medicine, Shanghai 200011, P.R. China

E-mail: guyan_8@hotmail.com

*Contributed equally

Key words: sulfatase 2, breast cancer, vascular endothelial growth factor-D, lymphangiogenesis, AKT1 modifying the sulfate patterns of heparan sulfate proteoglycans (HSPGs) located on the surface of most animal cells (1-3). HSPGs can be released into the extracellular matrix and can also be detected in serum. HSPGs carry out many structural and signaling functions through binding to protein ligands $(4,5)$. The Sulf family includes two structurally similar endogenous sulfatases (Sulf1 and Sulf2) with 64\% homology in highly conserved heparin-binding domains, but with different functions (2-4). Sulfatase 2 (Sulf2) is an extracellular endoglucosamine-6-sulfatase and considered as a bona fide cancer-causing agent in multiple types of cancer $(6,7)$. Sulf 2 is overexpressed in many tumor cells and was shown to promote tumorigenesis in many human cancers such as hepatocellular (8), pancreatic (9), ovarian $(10)$, breast $(6,10)$, and non-small cell lung carcinoma (11). Sulf2 also increased the activities of growth factors such as vascular endothelial growth factor (VEGF) and fibroblast growth factor-1 (FGF-1), and certain chemokines such as stromal cell-derived factor-1 (SDF-1) and secondary lymphoid-tissue chemokine (SLC), stimulating the biological functions of endothelial cells to promote angiogenesis $(5,12)$. Although Sulf2 was confirmed to facilitate angiogenesis, the effect of Sulf2 on lymphangiogenesis in tumors is still unknown.

Lymphangiogenesis, which is the formation of new lymphatic vessels, is a common process in normal tissue development, inflammation, wound healing and lymphatic edema $(13,14)$. Recently, more and more research has found lymphangiogenesis to play an important role in tumor progression and metastasis $(15,16)$. New lymphatic vessels are composed of one single layer of lymphatic endothelial cells. The basement membranes of new lymphatic vessels are incomplete, and the endothelial cells do not connect tightly. These factors allow tumor cells to easily invade new lymphatic vessels and metastasize to regional lymph nodes or distant organs (15-18). In recent years more research oncologists are becoming interested in the mechanisms of tumor-induced lymphangiogenesis in various tumors $(19,20)$. Breast cancer is one of the most common types of cancer among women worldwide $(21,22)$. Over $50 \%$ of early-stage breast cancer patients have local lymph node metastasis (18). Moreover, regional lymph node metastasis in breast cancer is also one of the main factors that leads to breast cancer metastasis and poor prognosis $(23,24)$. Tumor size and regional lymph node metastasis are used as 
biological indicators for breast cancer classification and selection markers for treatment strategies. Vascular endothelial growth factor-C (VEGF-C) and VEGF-D could combine with vascular endothelial growth factor receptor-3 (VEGFR-3) to induce lymphangiogenesis $(20,25,26)$. Our previous studies also suggested that breast cancer patients with high VEGF-D expression would have more regional lymph node metastasis, poor disease-free survival (DFS) and poor overall survival (OS) (20,25). Karpanen et al (24) demonstrated that when VEGF-D-overexpressing cells were implanted into transgenic mice, tumor-associated lymphangiogenesis was induced in several orthotopic mouse models.

Previously, we demonstrated that VEGF-D/FIGF, a member of the VEGF family, was upregulated by Sulf2 (6). In this study, we hypothesized that Sulf2 facilitates lymphangiogenesis in breast cancer by regulating VEGF-D. To evaluate the functions of Sulf2 in lymphangiogenesis in breast cancer, we examined the proliferation, apoptosis, cell cycle, mobility and tube-like structure formation of LECs in vitro, as well as lymphangiogenesis in mouse ears and xenografts in vivo. The expression of related signaling pathway genes was also screened and verified in LECs.

\section{Materials and methods}

Cell lines. Human breast cancer cell lines (MCF-7, MDA-MB-231) were purchased from the The Cell Bank of the Chinese Academy of Sciences (Shanghai, China). HEK293T cells used for lentivirus packaging were stocked in our own laboratory. All cells were cultured in Dulbecco's modified Eagle's medium (DMEM; HyClone Laboratories, Inc., Logan, UT, USA) with 10\% fetal bovine serum (FBS) and penicillin-streptomycin (all from Gibco, Grand Island, NY, USA). LECs were cultured in Endothelial Cell Growth Medium (ECGM) (both from PromoCell, Heidelberg, Germany). All cells were cultured at $37^{\circ} \mathrm{C}$ in $5 \% \mathrm{CO}_{2}$.

Conditioned medium (CM) collection. $\mathrm{CM}$ was collected from the supernatant of MCF-7 cells. MCF-7 cells release high levels of Sulf 2 protein in the supernatant which was confirmed in our previous study (6). The MCF-7 cells were cultured in DMEM until $80 \%$ confluence and were subsequently cultured in OptiMEM (HyClone Laboratories, Inc.) for another $72 \mathrm{~h}$. The supernatant was collected and concentrated using Amicon Ultra filters $30 \mathrm{D}$ (Millipore, Billerica, MA, USA) and then was kept in $50 \mathrm{mM}$ HEPES buffer ( $\mathrm{pH}$ 8.0; Biochrom $\mathrm{GmbH}$, Berlin, Germany) for further study.

Flag-Sulf2 vector construct. The signal peptide sequence of Sulf 2 was removed. The new peptide sequences of signal Flag and Ig-k were added with three rounds of PCR using three forward primers (Table I) and reverse primer 5'-CGG GATCCTTAACCTTCCCAGCCTTCCC-3'. The PCR conditions were $95^{\circ} \mathrm{C}$ for $5 \mathrm{~min}$, followed by 35 cycles of amplification, $95^{\circ} \mathrm{C}$ for $15 \mathrm{sec}, 55^{\circ} \mathrm{C}$ for $15 \mathrm{sec}$ and $72^{\circ} \mathrm{C}$ for $1 \mathrm{~min}$. The PCR sequence structures of flag-Sulf2 were signal peptide, signal peptide cleavage site, Flag, the linker portion of GSG and the Sulf 2 cDNA sequence (Table II). The amplified fragment was cloned into the pCDH (System Biosciences, Mountain View, CA, USA) to form the pCDH-Flag-Sulf2 vector construct. The sequences of the positive clone were identified using enzyme digestion and gene sequencing detection (Shanghai Meiji, Shanghai, China).

rSulf2 combination and purification. The pCDH-Flag-Sulf2 lentivirus was packaged in HEK $293 \mathrm{~F}$ cells using Lipofectamine 2000 (Invitrogen Life Technologies, Carlsbad, CA, USA) according to the manufacturer's instructions. The supernatant from the lentivirus-transfected HEK293F cells was collected and incubated with anti-Flag/M2 agarose beads (Sigma-Aldrich, St. Louis, MO, USA) at $4^{\circ} \mathrm{C}$ overnight. The beads were washed three times with washing buffer. The bound proteins were eluted with $0.1 \mathrm{mg} / \mathrm{ml}$ Flag peptide (DYKDDDDK). The eluate was concentrated though Amicon Ultra filters $30 \mathrm{D}$ and kept in $50 \mathrm{mM}$ HEPES buffer (pH 8.0).

$q R T-P C R$ analysis. Total RNA was isolated from cells using TRIzol reagent and reverse transcribed into cDNA using SuperScript III Reverse Transcriptase (both from Invitrogen Madison, WI, USA). The mRNA level was determined using the 7900HT qRT-PCR system (Applied Biosystems, Foster City, CA, USA) using SYBR ${ }^{\circledR}$ Green Real-time PCR Master Mix (Takara, Shiga, Japan). Primers for qRT-PCR are listed in Table III. Glyceraldehyde-3-phosphate dehydrogenase (GAPDH) was used as an internal control. Relative mRNA levels were calculated using the $\Delta \Delta \mathrm{Ct}$ method.

Western blot $(W B)$ analysis. Cells were harvested in the presence of a protease inhibitor cocktail (Sigma-Aldrich) in RIPA lysis buffer (Beyotime, Jiangsu, China). Equal amounts of proteins from the cells were resolved on SDS-PAGE and then transferred onto PVDF membranes as previously described (6). The membranes were separately probed with rabbit anti-FIGF (1:1,000, PAB4879; Abnova, Atlanta, GA, USA), rabbit anti-AKT1 (1:1,000, ab32505), rabbit anti-pAKT1 (s473) (1:1,000, ab66138; both from Abcam, Cambridge MA, USA), rabbit anti-mouse LYVE-1 (1:1,000, ab36993; AngioBio, San Diego, CA, USA), rabbit anti-GAPDH (1:500; Sigma-Aldrich) for $1 \mathrm{~h}$. Subsequently, the membranes were washed with TBST, and then incubated with goat anti-rabbit HRP (1:500; Sigma-Aldrich) for $1 \mathrm{~h}$. Bound antibody chemiluminescence was detected using chemiluminescence kits (Thermo Fisher Scientific, Darmstadt, Germany). The optical density was determined using a scanning densitometer and analyzed using Quantity One software (Bio-Rad, Hercules, CA, USA).

Proliferation assay. LECs were divided into four groups (control-1, rSulf2, VEGF-D, rSulf2+VEGF-D). Control-1 was cultured with only DMEM media. The other groups were separately cultured with rSulf2, VEGF-D and rSulf2+VEGF-D. The final concentration of rSulf 2 and VEGF-D in each group was $50 \mathrm{ng} / \mathrm{ml}$. The proliferation of LECs was assessed by the MTT method. Cells were dissociated from cell flasks by trypsin (Sigma-Aldrich) digestion and were seeded into 96-well plates $\left(1 \times 10^{5}\right.$ cells $\left./ \mathrm{ml}\right)$. The proliferation of LECs in the four groups was detected at different time-points $(0,12$, 24, 36 and $48 \mathrm{~h})$. All cells were incubated at $37^{\circ} \mathrm{C}$ for $4 \mathrm{~h}$ followed by the addition of $10 \mu \mathrm{l}$ MTT $(5 \mathrm{mg} / \mathrm{ml})$ and $100 \mu \mathrm{l}$ DMSO. The absorbance value of each well was measured 
Table I. Upstream primers of flag-Sulf2.

No. Primer sequences

\begin{tabular}{ll}
\hline Flag-Sulf2 F1 & 5'-GACGATTACAAGGATGACGACGATAAGGGTTCTGGCTTCCTGTCGCACCACCGC-3' \\
Flag-Sulf2 F2 & 5'-TGGGTACTGCTGCTCTGGGTTCCAGGTTCCACTGGTGACGATTACAAGGATGACGACG-3' \\
Flag-Sulf2 F3(Nhe I) & 5'-CTAGCTAGCATGGAGACAGACACACTCCTGCTATGGGTACTGCTGCTCTGG-3' \\
\hline
\end{tabular}

Table II. DNA sequences and amino acid sequences of flag-Sulf2.

\begin{tabular}{lll}
\hline Genes & \multicolumn{1}{c}{ DNA sequence } & Amino acid sequence \\
\hline Signal peptide & ATGGAGACAGACACACTCCTGCTATGGGTA & METDTLLLWVL \\
& CTGCTGCTCTGGGTTCCAGGTTCCACTGGT & LLWVPGST \\
Cleavage site & GAC & D \\
Flag & GATTACAAGGATGACGACGATAAG & DYKDDDDK \\
Linker & GGTTCTGGC & GSG \\
Sulf2 & TTCCTGTCGCACCACCGCCTGAAA... & FLSHHRLK... \\
& (Sulf2 full length...) & (Sulf2 full length \\
& & amino acid chain...) \\
\hline
\end{tabular}

Table III. Real-time PCR primers.

\begin{tabular}{ll}
\hline Gene & \multicolumn{1}{c}{ Primer sequences } \\
\hline GAPDH (HUMAN) & F 5'-GGGAAACTGTGGCGTGAT-3' \\
PLA2G1B & R 5'-GAGTGGGTGTCGCTGTTGA-3' \\
& F 5'-TGTGGCAGTTCCGCAAAAT-3' \\
PLA2G5 & R 5'-GCAGCCGTAGTTGTTGTATTCC-3' \\
& F 5'-AACCCCAGAGATGAAAGGC-3' \\
PLA2G6 & R 5'-CGTAGTTTGTCAGGGCGTTC-3' \\
& F 5'-CCACATCATCCCTTCTCCCT-3' \\
PLA2G2D & R 5'-CTTTCACTCCTCCTCCATCCA-3' \\
AKT1 & F 5'-GGCCTAGAGTGGCAAATGG-3' \\
& R 5'-GGGAAAACAGGGGAAACAGA-3' \\
PIK3R1 & F 5'-GCCCTGCTACCTGTTCTTGG-3' \\
& R 5'-AAGCAAATGGCAAAGTGTGAG-3' \\
PIK3R3 & F 5'-TTGGAAGCAGCAACCGAAAC-3' \\
& R 5'-CTTCGCCGTCCACCACTACA-3' \\
& F 5'-TGGTTCAGCACAACGACTCC-3' \\
& R 5'-CACCTCTCTTCCCACTTCCT-3'
\end{tabular}

F, forward; $R$, reverse.

using a microplate reader (Bio-Tek, Winooski, VT, USA) at a wavelength of $570 \mathrm{~nm}$.

Apoptosis assay. The aforementioned four groups were used. Apoptosis was determined by dual staining using Annexin V/FITC and propidium iodide (Invitrogen). Briefly, the $\log$ phase of LECs was seeded into 24-well cell culture plates $\left(1 \times 10^{5}\right.$ cells/well). Subsequently, LECs were treated with $10 \mu \mathrm{g} / \mathrm{ml}$ cisplatin (Qilu Pharmaceutical Co., Shanghai, China) for $24 \mathrm{~h}$ and then dissociated from the wells with $0.25 \%$ trypsin, spun at $1,500 \mathrm{rpm}$ for $5 \mathrm{~min}$, resuspended in Annexin V binding buffer, stained with $1 \mu \mathrm{l}$ Annexin V/ FITC for $15 \mathrm{~min}$ and $1 \mu \mathrm{l}$ propidium iodide for $1 \mathrm{~min}$. The cells were analyzed using the FACSCalibur System (BD Biosciences, San Jose, CA, USA). The relative proportion of Annexin V-positive cells, representing apoptotic cells, was 
determined using FlowJo software (FlowJo, LLC, Ashland, OR, USA).

Cell cycle assay. The aforementioned four groups were used. The cell cycle distribution of LECs was determined by propidium iodide staining and flow cytometric analysis. After LECs were treated with $10 \mu \mathrm{g} / \mathrm{ml}$ cisplatin for $24 \mathrm{~h}$, the cells were dissociated from the wells with $0.25 \%$ trypsin. Subsequently, they were fixed in $70 \%$ ethanol overnight at $-20^{\circ} \mathrm{C}$ and incubated in RNase A at $37^{\circ} \mathrm{C}$ for 30 min. Propidium iodide was then added and the cells were incubated in a dark room for $30 \mathrm{~min}$. Flow cytometry was used to detect the cell cycle distribution. The proliferation index (PI) was calculated using the formula PI $=(S+G 2) /(S+G 1+G 2) \times 100 \%$.

LEC mobility assay. The aforementioned four groups were used. The mobility of LECs was determined in 12-well Boyden chamber plates and polycarbonate membrane filter inserts (CoStar Group, Inc., Washington, DC, USA) with $8-\mu \mathrm{m}$ pores. For the cell mobility assay, the interior of the Transwell insert was coated with Matrigel (BD Pharmingen, San Diego, CA, USA), which mimics the basement membrane. In all, $1 \times 10^{5}$ cells were seeded into the upper chamber. The cell suspension was also seeded onto the membrane in the upper chamber and the lower chamber was filled with $1 \mathrm{ml}$ medium with $10 \%$ FBS. After $48 \mathrm{~h}$, the non-migrating cells on the surface of the upper chamber were removed with cotton swabs. The migrating cells at the bottom of the membrane were fixed in formaldehyde for $1 \mathrm{~min}$ and then stained with crystal violet. The stained membranes were cut and placed onto a glass slide and the number of invading cells at the bottom surface of the membrane was counted three times under a bright-field light microscope.

Lymphatic tube-like structure formation assay. The aforementioned four groups were used. The lymphangiogenic capacities of LECs on Matrigel were determined according to the manufacturer's instructions. The Matrigel was melted in $4^{\circ} \mathrm{C}$ and was diluted to half its concentration by media and then was added into 24-well plates for cooling down. The 24-well plates were placed in an incubator for $30 \mathrm{~min}$ to solidify the glue. LECs $\left(5 \times 10^{5}\right)$ were digested and added into each well before this solidification process. The numbers of new lymphatic tubes were detected using an inverted phase contrast microscope (AMG, Bovenden, Germany) after $24 \mathrm{~h}$ of culture.

Lymphangiogenesis in mouse ears. Four-week-old, BALB/ c-nu mice (weight, $15 \mathrm{~g}$ ) were purchased from the Shanghai Experimental Animal Center of the Chinese Academy of Sciences (Shanghai, China). The mice were divided into three groups (control-2, CM-nu, rSulf2-nu). A total of $0.1 \mathrm{ml}$ $0.9 \%$ saline, CM $(50 \mathrm{mM})$ and rSulf $2(50 \mathrm{mM})$ were separately injected into the root of the mouse ears every day for six weeks. Excised mouse ears were fixed in formalin buffer and embedded in paraffin for advanced testing. All experimental protocols followed the instructions of the Chinese Council on Animal Care and were approved by the Animal Experimental Ethical Inspection of Shanghai Ninth People's Hospital affiliated to Shanghai Jiaotong University, School of Medicine [permit (no. 20015) 25].

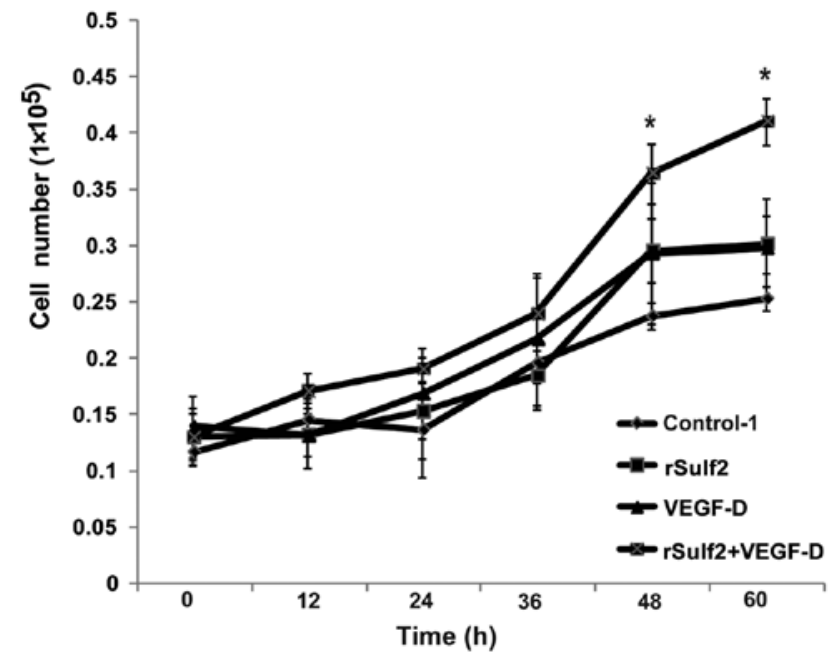

Figure 1. Growth curve of lymphatic endothelial cells (LECs) at different time-points. Cell growth curves were drawn based on the absorbance value of live cells at different time-points. Compared with the control-1, LECs treated with recombinant Sulf2 (rSulf2) or vascular endothelial growth factor-D (VEGF-D) showed higher cell proliferation after $48 \mathrm{~h}$, but the difference was not significant. However, the rSulf2+VEGF-D group showed significantly higher cell proliferation than control-1 at 48 and $60 \mathrm{~h},{ }^{*} \mathrm{P}<0.05$.

Lymphangiogenesis in breast cancer xenografts. Six-week-old, 18-g female Nod/scid mice were purchased from Shanghai Experimental Animal Center of the Chinese Academy of Sciences. MDA-MB-231 breast cancer cells were detached with $0.25 \%$ trypsin and resuspended in HBSS/Matrigel (1:1 volume) to $10^{7}$ cells $/ \mathrm{ml}$. Xenografts were generated by injecting $0.2 \mathrm{ml}$ cell suspension into the area of the mammary fat pad. Mice were divided into three groups (control-3, CM-scid, rSulf2-scid). A total of $0.1 \mathrm{ml} 0.9 \%$ saline, CM $(50 \mathrm{mM})$ and rSulf2 $(50 \mathrm{mM})$ were separately injected into xenografts every day until 6 weeks. Excised xenografts were fixed in formalin buffer and embedded in paraffin for advanced testing. All experimental protocols followed the instructions of the Chinese Council on Animal Care and were approved by the Animal Experimental Ethical Inspection of Shanghai Ninth People's Hospital affiliated to Shanghai Jiaotong University, School of Medicine [permit (no. 20015) 25].

Immunohistochemistry (IHC). Five-micron-thick sections of the paraffin-embedded tissues were deparaffinized in xylenes and rehydrated through a graded alcohol series. Heat-induced epitope retrieval (HIER) was performed by immersion of the tissue sections in $8 \mathrm{mM}$ EDTA buffer ( $\mathrm{pH} \mathrm{8.0)}$ for $20 \mathrm{~min}$ at $98^{\circ} \mathrm{C}$. IHC staining was performed using a horseradish peroxidase-labeled polymer K4001 (Dako, Zagreb, Croatia) according to the manufacturer's instructions. Briefly, the slides were incubated with $3 \%$ hydrogen peroxide and $1 \%$ bovine serum albumin (BSA; Sigma-Aldrich) for $10 \mathrm{~min}$ each. To visualize lymphatic vessels, the sections were exposed to the primary antibody, rabbit anti-mouse LYVE-1 which was diluted as recommended in $3 \% \mathrm{BSA}$, for $1 \mathrm{~h}$ at room temperature. The slides were then incubated with goat anti-rabbit HRP for 30 min followed by incubation with the DAB chromogen (Dako) for 5 min. Finally, the slides were counterstained with hematoxylin (Thermo Fisher Scientific, Waltham, MA, USA), blued in $1 \%$ ammonium hydroxide, 
A

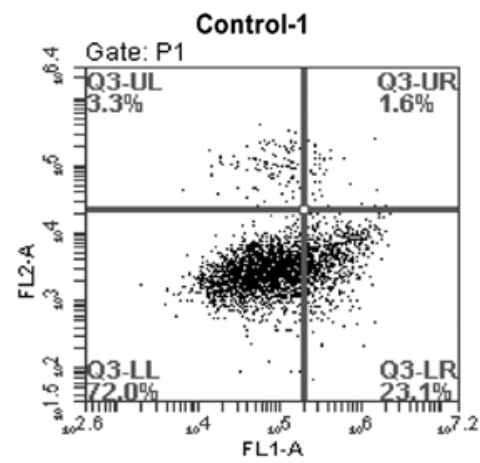

VEGF-D

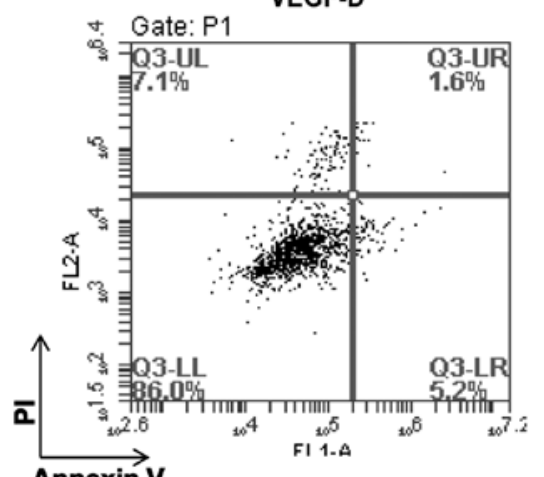

$\underset{\text { Annexin V }}{\longrightarrow}$

B

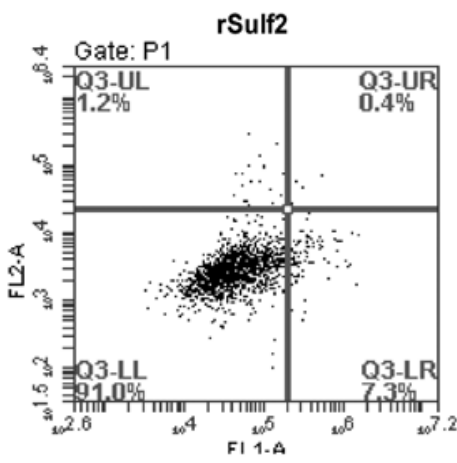

rSulf2+VEGF-D

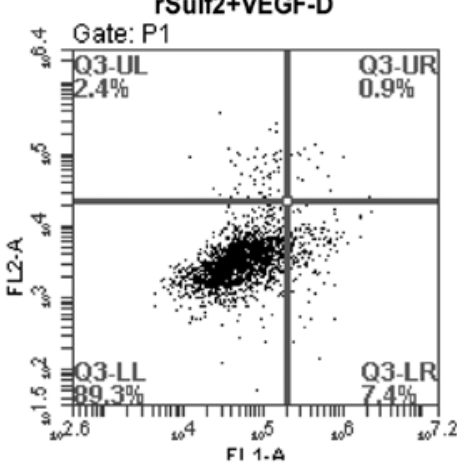

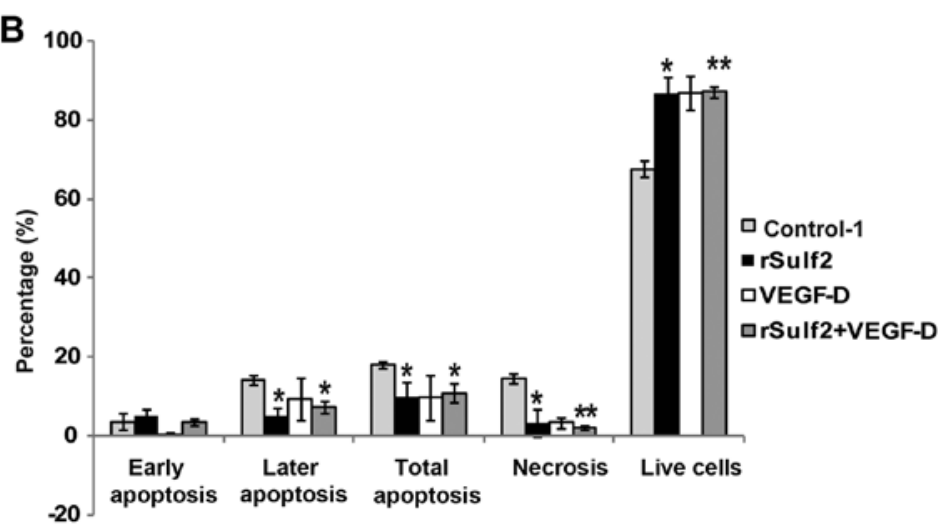

Figure 2. The apoptosis of lymphatic endothelial cells (LECs) detected by flow cytometry. (A) Annexin V/propidium iodide-labeled apoptotic LECs were detected using flow cytometry. (B) The effects of recombinant Sulf2 (rSulf2), vascular endothelial growth factor-D (VEGF-D) and rSulf2+VEGF-D on LEC apoptosis. ${ }^{*} \mathrm{P}<0.05,{ }^{* *} \mathrm{P}<0.01$.

dehydrated, and mounted with Acrymount. Consecutive sections where the primary antibody was omitted were used as negative controls. The washing buffer used was $1 \mathrm{X}$ TBS with $0.05 \%$ Tween-20 (Thermo Fisher Scientific).

Statistical analysis. Data are presented as the mean \pm standard deviation (SD) of at least three independent experiments with three or more replicates. Continuous data were analyzed using a two-tailed Student's t-test. $\mathrm{P}<0.05$ was considered to indicate a significant difference.

\section{Results}

Sulf2 with VEGF-D promotes LEC proliferation. To evaluate the role of Sulf2 in LEC proliferation, an MTT assay was used to detect the proliferation of LECs at different time-points. Cell growth curves were drawn based on the absorbance value of live cells at different time-points. Compared with the control-1, the rSulf2 and VEGF-D groups showed higher cell growth after $36 \mathrm{~h}$, but the difference was not significant. However, the group treated with rSulf2+VEGF-D showed a significant difference in the absorbance of live cells at 48 and $60 \mathrm{~h}(0.36 \pm 0.03$ vs. $0.24 \pm 0.01,0.41 \pm 0.02$ vs. $0.25 \pm 0.01$ respectively, $\mathrm{P}<0.05$, Fig. 1). The results indicated that Sulf2 or VEGF-D could enhance LEC proliferation, but their effects were not significant. Furthermore, LECs treated with rSulf2 and VEGF-D showed a significantly higher growth rate than the cells treated with control-1. Collectively, these data indicated that Sulf 2 could promote breast cancer proliferation through the activation of VEGF-D.

Sulf2 inhibits cisplatin-induced LEC apoptosis. To evaluate the role of Sulf2 in LEC apoptosis, we measured the cisplatin-induced apoptosis and necrosis of LECs by flow cytometry. Compared with the control-1, treatment with rSulf 2 resulted in a significant increase in the percentage of 
A
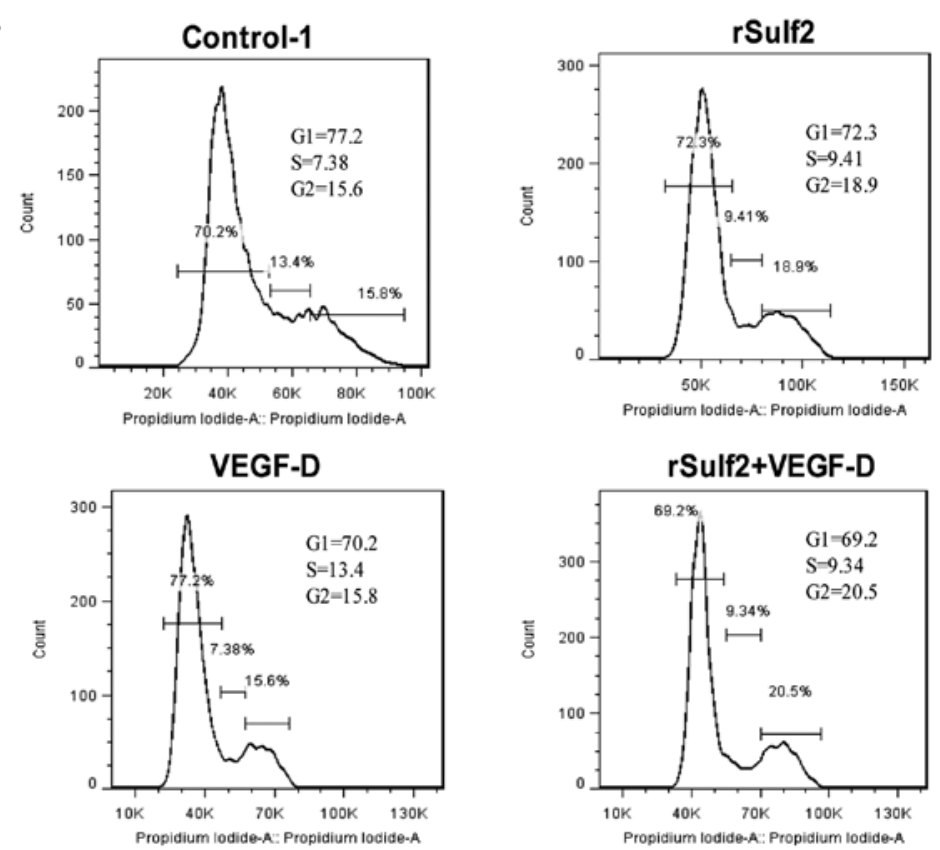

B

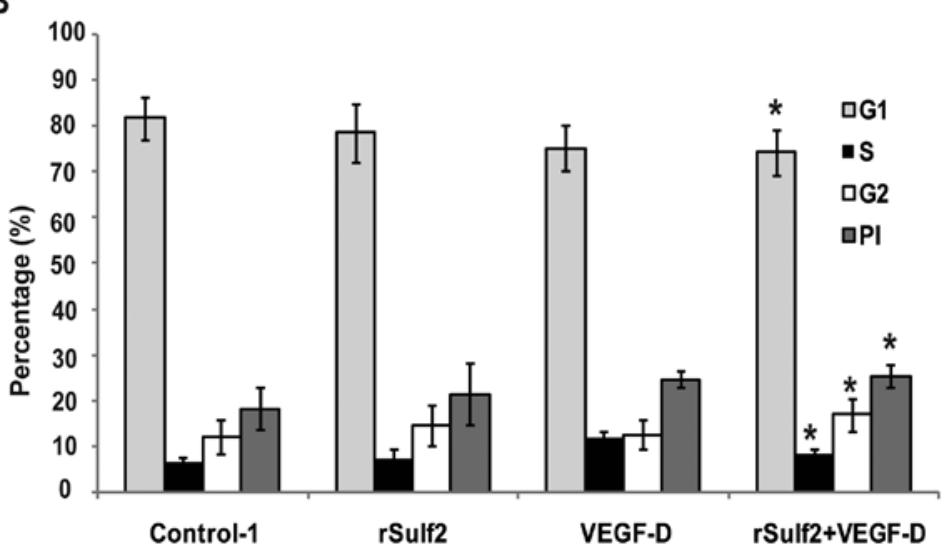

Figure 3. Cell cycle distribution of lymphatic endothelial cells (LECs) as detected by flow cytometry. (A) Cell cycle distribution in the LECs was detected using flow cytometry, and the abscissa indicated the amount of DNA. The G1, S and G2/M phases are marked. (B) Effects of recombinant Sulf2 (rSulf2), vascular endothelial growth factor-D (VEGF-D) and rSulf2+VEGF-D on the cell cycle of LECs. "P<0.05.

live cells $(86.98 \pm 3.84$ vs. $67.60 \pm 2.12, \mathrm{P}<0.05)$ and a significant decrease in total apoptosis $(9.75 \pm 4.03$ vs. $17.95 \pm 0.78, \mathrm{P}<0.05)$. A closer look at the different stages in apoptosis revealed that the most significant difference occured in the late stage $(4.95 \pm 2.19$ vs. $14.3 \pm 1.27, \mathrm{P}<0.05)$ instead of the early stage $(4.80 \pm 1.83$ vs. $3.65 \pm 2.05, \mathrm{P}>0.05)$. Treatment with VEGF-D caused a significant decrease in the percentage of dead cells ( $3.35 \pm 1.48$ vs. $14.5 \pm 1.27, \mathrm{P}<0.05)$, but had no significant effects on the percentage of live cells $(86.75 \pm 4.31$ vs. $67.6 \pm 2.12$, $\mathrm{P}>0.05)$ and total apoptosis $(9.85 \pm 5.72$ vs. $17.95 \pm 0.78$, $\mathrm{P}>0.05)$. Treatment with rSulf2+VEGF-D resulted in a significant increase in the percentage of live cells $(87.11 \pm 1.27$ vs. $67.60 \pm 2.12, \mathrm{P}<0.01)$ and a more significant decrease in total apoptosis $(10.81 \pm 2.40$ vs. $17.95 \pm 0.78, \mathrm{P}<0.05)$ and percentage of dead cells $(2.05 \pm 0.64$ vs. $14.50 \pm 1.27$, $\mathrm{P}<0.01)$, especially in the late stage $(7.31 \pm 1.56$ vs. $14.31 \pm 1.27$, $\mathrm{P}<0.05$ ) (Fig. 2A and B). The results showed that VEGF-D had no direct effect on cisplatin-induced LEC apoptosis. The rSulf2- and rSulf2+VEGF-D-treated groups showed a significant increase in the percentage of live cells, decreased cell necrosis and inhibited cisplatin-induced LEC apoptosis, particularly in the late stage of apoptosis. However, rSulf2+VEGF-D treatment had a greater effect on apoptosis. Based on these results, rSulf2 inhibited the apoptosis of LECs by activating VEGF-D.

Sulf2 with VEGF-D improves cell cycle distibution of cisplatin-pretreated LECs. To ascertain the role of Sulf2 in the cell cycle control of LECs, the cell cycle distribution of LECs was assessed by flow cytometry. Compared with the control-1, rSulf2 treatment caused no difference in the number of cells in the G1 phase ( $78.70 \pm 6.40$ vs. $81.75 \pm 4.55, \mathrm{P}>0.05)$, the $S$ phase $(6.99 \pm 2.42$ vs. $6.31 \pm 1.08, P>0.05)$ and the $G 2 / M$ phase $(14.45 \pm 4.45$ vs. $11.99 \pm 3.61, \mathrm{P}>0.05)$. Moreover, it had no effect on the PI index $(21.41 \pm 6.76$ vs. $18.29 \pm 4.65, \mathrm{P}>0.05)$. Furthermore, VEGF-D treatment had no significant effect on the PI index $(24.55 \pm 3.23$ vs. $18.29 \pm 4.65, \mathrm{P}>0.05)$, the number of cells in the G1 phase $(75.23 \pm 5.03$ vs. $81.75 \pm 4.55, \mathrm{P}>0.05)$, the $S$ phase $(11.9 \pm 1.5$ vs. $6.31 \pm 1.08, P>0.05)$ and the $G 2 / M$ phase $(12.56 \pm 3.26$ vs. $11.99 \pm 3.61, \mathrm{P}>0.05)$. rSulf2+VEGF-D 
A

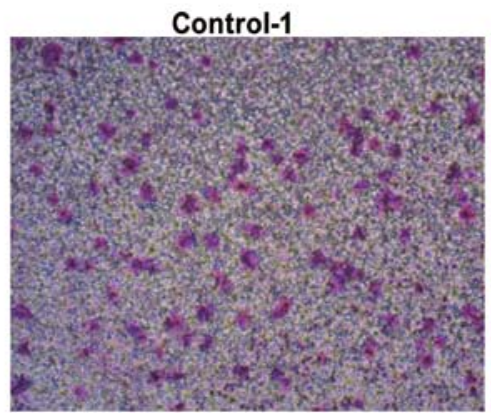

VEGF-D

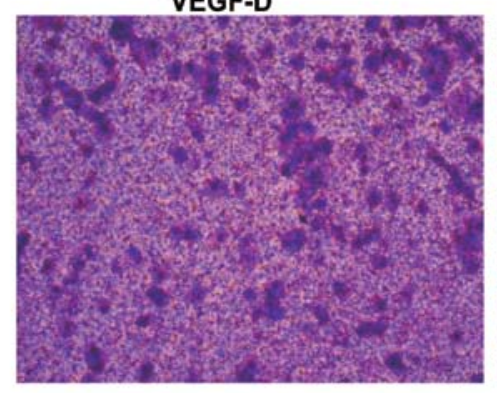

rSulf2

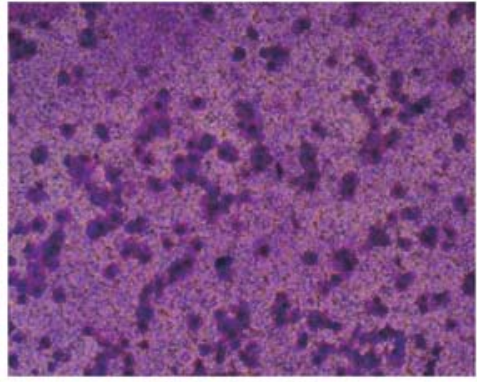

rSulf2+VEGF-D

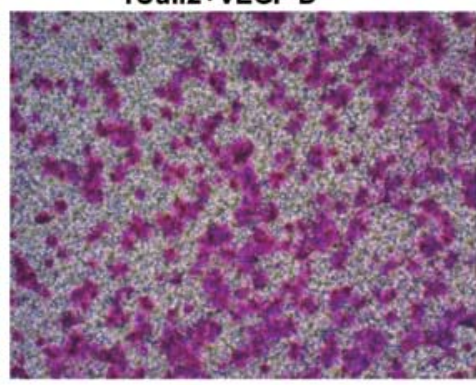

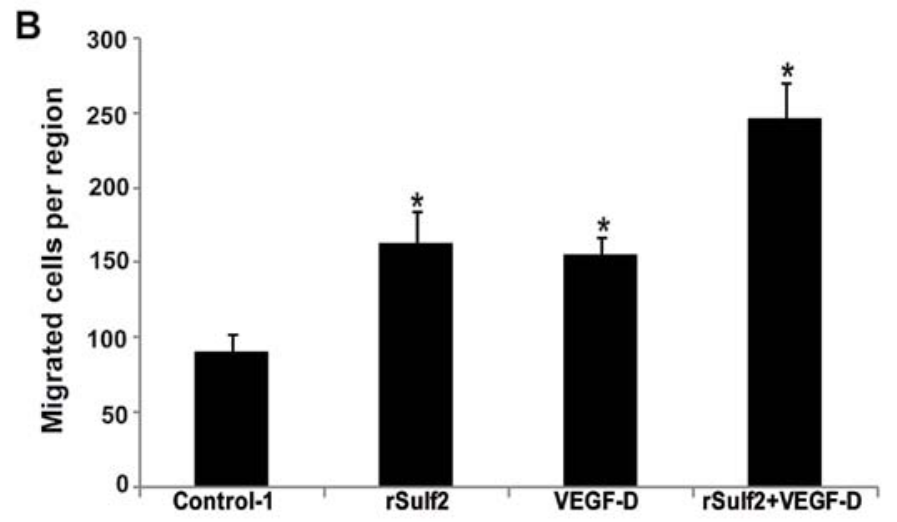

Figure 4. Lymphatic endothelial cell (LEC) mobility assay using a Transwell chamber. (A) LECs migrated to the lower side of the chamber membranes after being incubated for $48 \mathrm{~h}$. (B) The number of LECs that migrated though the chamber membranes. "P<0.05.

Control-1

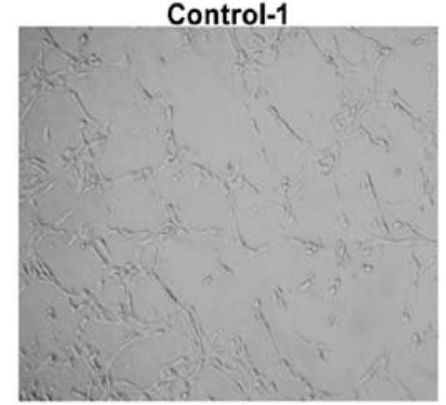

VEGF-D

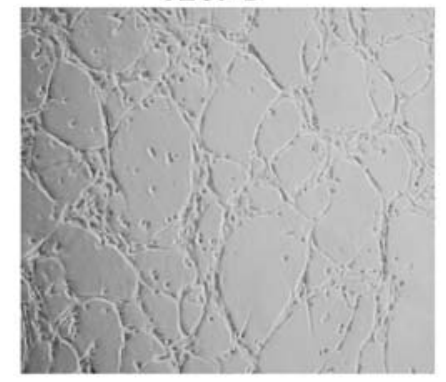

rSulf2

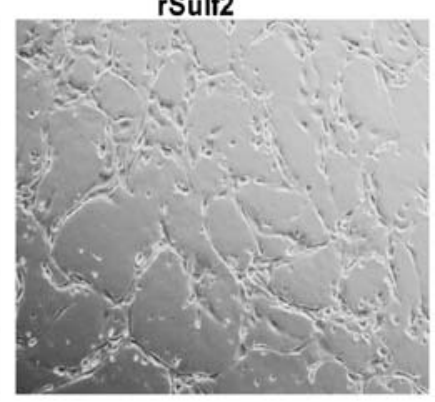

rSulf2+VEGF-D

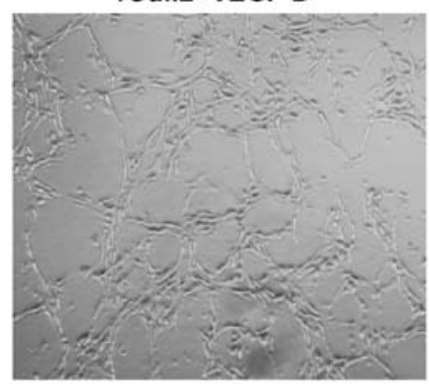

treatment caused a significant decrease in the number of cells in the $\mathrm{G} 1$ phase $(74.3 \pm 5.10$ vs. $81.75 \pm 4.55, \mathrm{P}<0.05)$, the $\mathrm{S}$ phase $(8.32 \pm 1.02$ vs. $6.31 \pm 1.08, \mathrm{P}<0.05)$ and the $\mathrm{G} 2 / \mathrm{M}$ phase $(16.95 \pm 3.55$ vs. $11.99 \pm 3.61, \mathrm{P}<0.05)$ as well as a significantly higher PI index $(25.37 \pm 2.50$ vs. 18.29 \pm 4.65 , $\mathrm{P}<0.05)$. We concluded that Sulf2 together with VEGF-D significantly promoted cell cycle progression from the G1 phase to the G2/M phase and increased the PI index in the LECs (Fig. 3A and B), while Sulf2 or VEGF-D alone had no significant effect on the cell cycle of the LECs.

Sulf 2 promotes breast cancer migration. Compared with the control-1, the rSulf2- or VEGF-D-treated LECs showed higher migration through the membrane of the Boyden

Figure 5. The effect of recombinant Sulf2 (rSulf2) in an in vitro lymphatic tube formation assay. Compared with control-1, treatment with rSulf2, vascular endothelial growth factor-D (VEGF-D) and rSulf2+VEGF-D resulted in a significant increase in the number of new lymphatic tubes formed, especially in the rSulf2+VEGF-D group. 


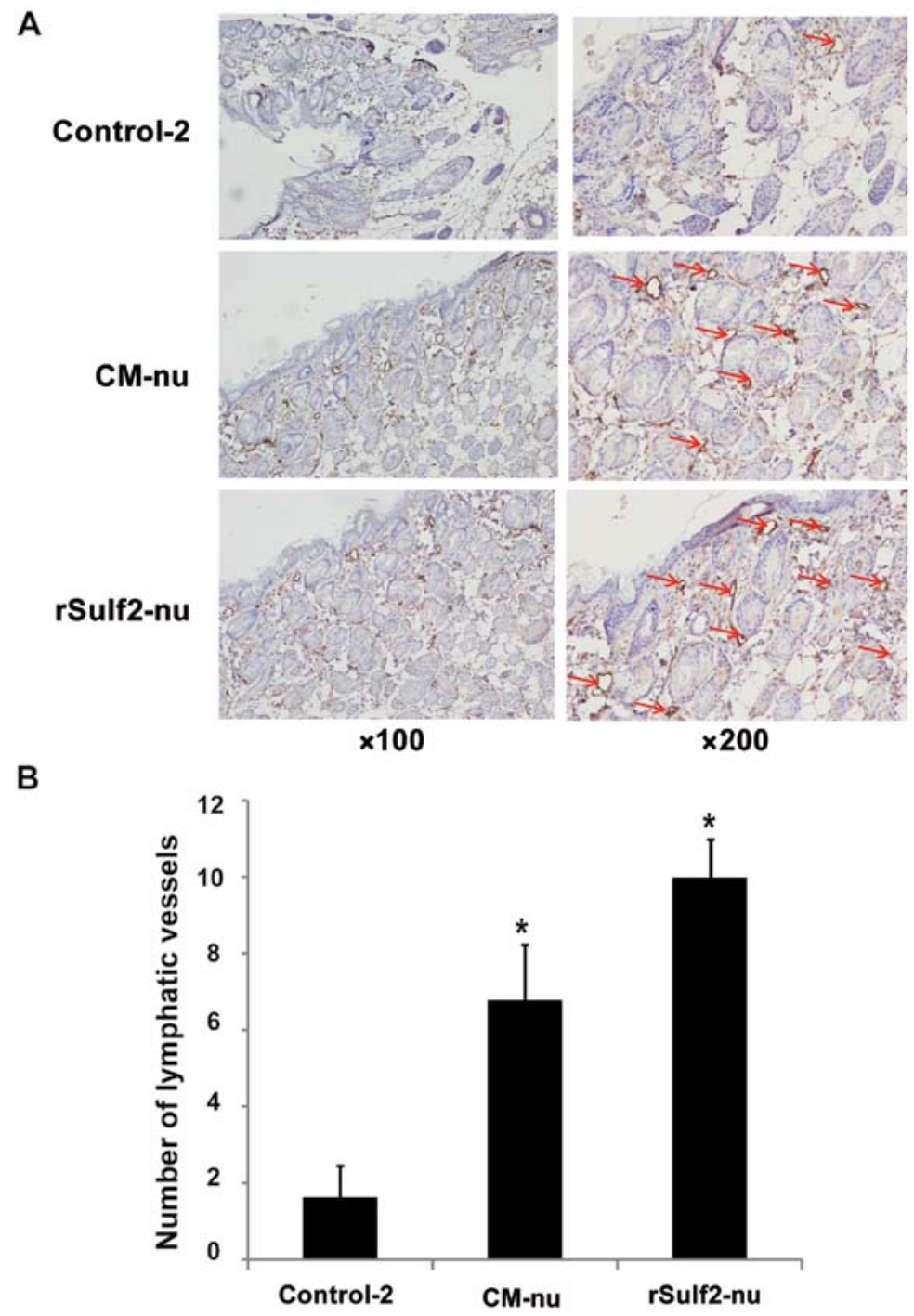

Figure 6. Lymphatic vessels in nude mouse ears detected by immunohistochemical staining. (A) Immunohistochemical staining showing the number of lymphatic vessels in nude mouse ears are indicated by the red arrows. (B) Quantification of the number of lymphatic vessels in the three groups, ${ }^{*} \mathrm{P}<0.05$.

chamber $(163.33 \pm 20.98,155.67 \pm 10.96$ vs. $90.0 \pm 12.52$, $\mathrm{P}<0.05)$. Moreover, the rSulf2+VEGF-D treated cells showed the highest migration rate $(247.33 \pm 23.07$ vs. $90.0 \pm 12.52$, $\mathrm{P}<0.05)$ (Fig. 4A and B). These observations clearly suggested that rSulf2 or VEGF-D enhanced LEC migration, but rSulf2 with VEGF-D might work synergistically.

Sulf 2 promotes lymphatic tube-like structure formation in vitro. To examine the effect of Sulf 2 on lymphatic tube-like structure formation of LECs in vitro, LECs were seeded on Matrigel substrate. Compared with the control-1, more lymphatic tube-like structures were formed by LECs treated with rSulf2, VEGF-D, and rSulf2+VEGF-D, after $24 \mathrm{~h}$ (Fig. 5). The results showed that rSulf2 or VEGF-D increased lymphatic tube-like structure formation of the LECs, however, the effect of Sulf2 with VEGF-D was more significant, suggesting that Sulf 2 could promote lymphangiogenesis in vitro through the activation of VEGF-D.

Sulf2 improves lymphangiogenesis in nude mouse ears. The nude mouse ears were examined by pathological sections.
The lymphatic vessels were detected using IHC. Compared with the control-2, the CM-nu and rSulf2-nu groups showed significantly more lymphatic vessels $(6.8 \pm 1.48$ vs. $1.6 \pm 0.89$, $\mathrm{P}<0.01,10 \pm 1.00$ vs. $1.6 \pm 0.89, \mathrm{P}<0.05$ ). Furthermore, rSulf2-nu also showed more lymphatic vessels compared with the CM-nu group $(10 \pm 1.00$ vs. $6.8 \pm 1.48, \mathrm{P}<0.05)$. The results demonstrated that both exogenous and endogenous Sulf 2 from breast cancer xenografts promoted lymphangiogenesis in nude mouse ears. Moreover, the effects of purified exogenous Sulf2 on lymphangiogenesis were more pronounced than endogenous Sulf2 (Fig. 6).

Sulf 2 promotes lymphangiogenesis in the breast cancer xenografts. To detect the effect of Sulf 2 on lymphangiogenesis in the breast cancer xenografts, we detected the density of lymphatic vessels in MDA-MB-231 breast cancer xenografts, which did not express Sulf2. No significant lymphatic vessels were detected inside or around the xenografts in control-3. More lymphatic vessels around the xenografts were detected in the CM-scid and rSulf2-scid groups (Fig. 7). The results further certified that Sulf2 increased lymphangiogenesis in 


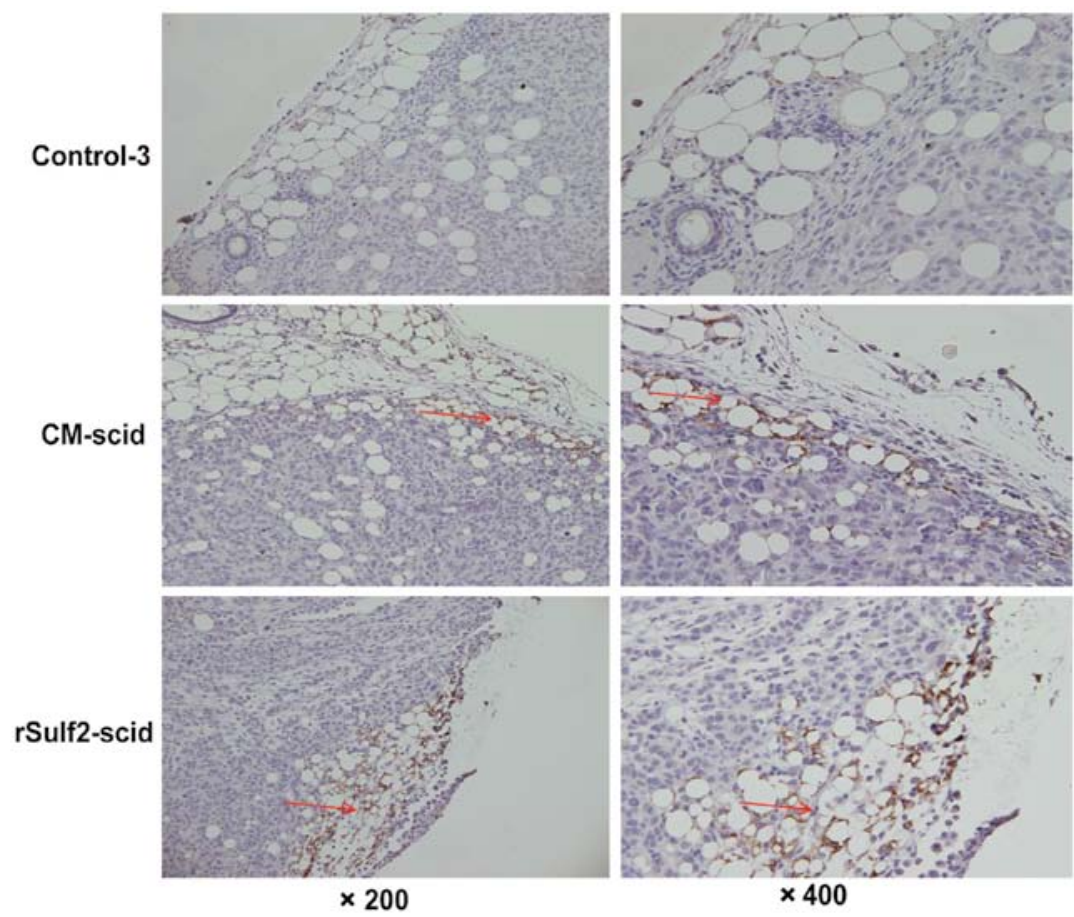

Figure 7. Sulfatase 2 (Sulf2) promotes lymphangiogenesis in the breast cancer xenografts. The images show lymphatic vessels in the breast cancer xenografts, which are marked by red arrows. No lymphatic vessels were detected inside or around the xenografts in control-3. Conditioned medium (CM)-scid and recombinant Sulf2 (rSulf2)-scid groups showed more lymphatic vessels around the xenografts.

A

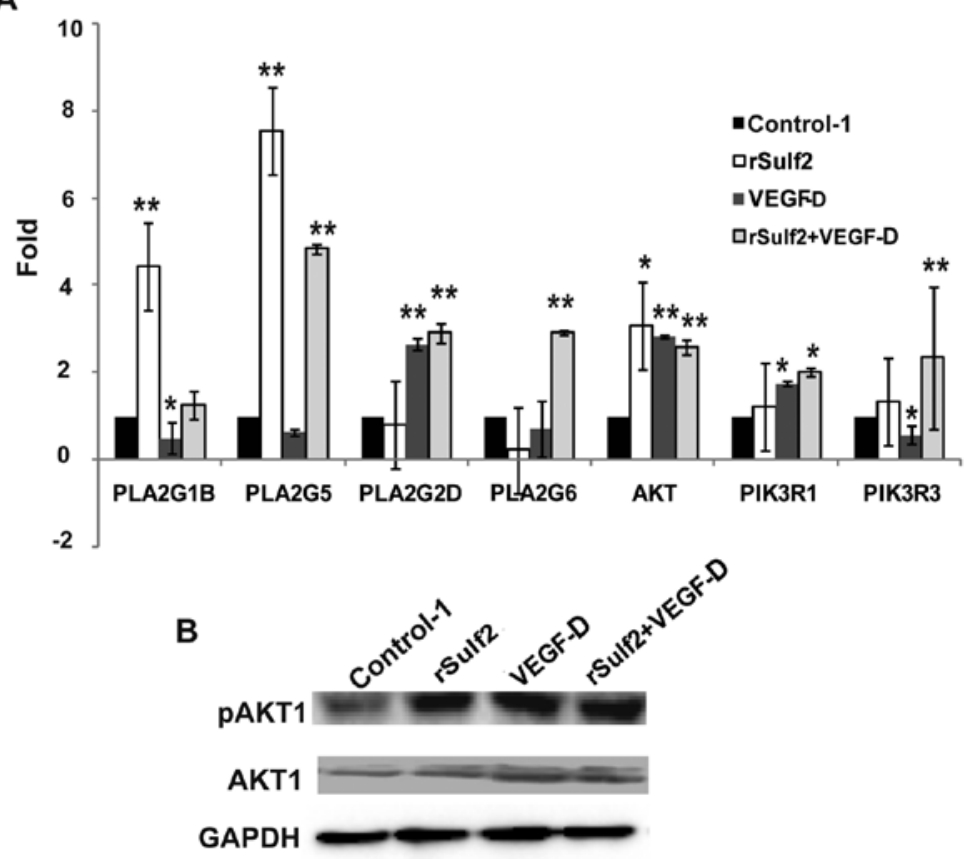

Figure 8. Change in gene expression caused by Sulfatase 2 (Sulf2) with/without vascular endothelial growth factor-D (VEGF-D) treatment. (A) Gene expression change in lymphatic endothelial cells (LECs) verified by qRT-PCR. (B) The AKT1 protein expression and phosphorylation were affected by Sulf2 and VEGF-D treatment. ${ }^{*} \mathrm{P}<0.05,{ }^{* *} \mathrm{P}<0.01$.

breast cancer xenografts and that breast cancer cells secreted Sulf2 to promote lymphangiogenesis.

Sulf2 regulates signaling pathway molecular interactions in LECs. Messenger RNA levels of a panel of VEGF signaling pathway genes were first analyzed by PCR microarray, followed by qRT-PCR and WB analysis verification. Compared with the control-1, the genes significantly upregulated following treatment with rSulf2 were PLA2G1B (4.44 \pm 0.84 vs. $1.00, \mathrm{P}<0.01)$, PLA2G5 $(7.54 \pm 1.21$ vs. 1.00 , $\mathrm{P}<0.01)$ and AKT1 $(3.09 \pm 0.62$ vs. $1.00, \mathrm{P}<0.05)$. The genes significantly upregulated by VEGF-D were PLA2G2D 
(2.67 \pm 0.14 vs. $1.00, \mathrm{P}<0.01)$, AKT1 (2.85 \pm 0.04 vs. $1.00, \mathrm{P}<0.01)$ and PI3KR1 $(1.76 \pm 0.06$ vs. $1.00, \mathrm{P}<0.05)$. The genes downregulated were PLA2G1B $(0.49 \pm 0.36$ vs. $1.00, \mathrm{P}<0.05)$ and PI3KR3 $(0.57 \pm 0.21$ vs. $1.00, \mathrm{P}<0.05)$. The genes significantly upregulated in the rSulf2+VEGF-D group were PLA2G5 $(4.84 \pm 0.12$ vs. $1.00, \mathrm{P}<0.01)$, PLA2G2D $(2.91 \pm 0.21$ vs. 1.00 , $\mathrm{P}<0.01)$, PLA2G6 $(2.93 \pm 0.04$ vs. $1.00, \mathrm{P}<0.01)$, AKT1 $(2.59 \pm 0.16$ vs. $1.00, \mathrm{P}<0.01)$ and PI3KR1 $(2.01 \pm 0.1$ vs. 1.00 , $\mathrm{P}<0.01$ ) (Fig. 8A). Only AKT1 mRNA showed the same significant trends in the three groups and was chosen for further WB analysis verification. Furthermore, we tested the AKT1 and the phosphorylated AKT1 protein by WB analysis in the four groups. Compared with the control-1, the expression and phosphorylation of AKT1 in the LECs revealed a significant increase in the other groups (Fig. 8B). The results revealed that Sulf2 and/or VEGF-D could promote AKT1 expression and activation in the LECs.

\section{Discussion}

Sulf2 has been reported to modify the activities of heparan-binding growth factors (VEGF and FGF-1) and influence the signaling pathways of the corresponding receptors to facilitate angiogenesis. Uchimura et al (5) validated Sulf2 as a new molecule involved in angiogenesis through the activation of VEGF and FGF-1. Skobe et al (19) and Cherng et al (26) certified that the VEGF family is comprised of different monomeric forms including VEGF145, VEGF165 and VEGF189 and VEGF206. These different monomeric forms had similar heparan-binding regions, which could be regulated by Sulf2. VEGF-D is one member of the VEGF family and also shares similar structures. Harris et al (27) reported that VEGF-D is an angiogenic and lymphangiogenic glycoprotein. Heparan-binding regions in VEGF-D were found within the $\mathrm{N}$ - and $\mathrm{C}$-terminal propeptides, which suggested that VEGF-D could also bind to heparan. The C-terminal propeptide significantly enhanced this interaction through the removal of this propeptide from full-length VEGF-D. The removal of either the $\mathrm{N}$ - or $\mathrm{C}$-terminal propeptide was required for VEGF-D binding to VEGFR-2/VEGFR-3 and formation of heterodimers, which have recently been shown to positively regulate angiogenic and lymphangiogenic sprouting $(28,29)$. In contrast, the removal of both propeptides was required for high rates of lymph node metastasis. It was also reported that the propeptides profoundly influenced the molecular interactions of VEGF-D with VEGFR-3, and these propeptide structures also promoted the effects of VEGF-D on tumor development.

In our previous study, we demonstrated that Sulf2 was upstream of VEGF-D and upregulated VEGF-D expression in breast cancer cells (6). In this study, we studied the role of Sulf 2 in lymphangiogenesis and the mechanism involved in its function. MCF-7 breast cancer cells released a high level of Sulf2 protein into the culture medium, which was demonstrated in our previous study (6). In this study, we collected the CM from the supernatant of MCF-7 cells to study the effect of endogenous Sulf2 on lymphangiogenesis in breast cancer cells. We also combined and purified exogenous rSulf 2 to study the direct function and mechanism of Sulf2 in lymphangiogenesis. We found that Sulf2 significantly increased LEC mobility and lymphatic tube-like structure formation, inhibited cisplatin-induced LEC apoptosis in vitro, but had no direct effect on cell proliferation and the cell cycle. Moreover, rSulf2 together with VEGF-D, further promoted the proliferation, cell cycle progression, mobility and tube formation in LECs, while at the same time inhibited cisplatin-induced apoptosis, especially in the late stage. Sulf2 also significantly improved the densities of lymphatic vessels in mouse ears and breast cancer xenografts in vivo. These results showed that Sulf2 not only enhanced VEGF-D expression, but also enhanced the activity of VEGF-D. Furthermore, we found that the signaling pathway gene AKT1 was upregulated and activated by Sulf2.

In summary, Sulf2 markedly promoted lymphangiogenesis in breast cancer, possibly by promoting VEGF-D expression and by activating the AKT1-related signaling pathway. This finding confirmed the role of Sulf2 as a biomarker of breast cancer progression. More importantly, new therapeutic approaches targeting Sulf 2 could improve the clinical outcomes in patients with lymph node-positive breast cancer.

\section{References}

1. Bishop JR, Schuksz M and Esko JD: Heparan sulphate proteoglycans fine-tune mammalian physiology. Nature 446: 1030-1037, 2007.

2. Rosen SD and Lemjabbar-Alaoui H: Sulf-2: An extracellular modulator of cell signaling and a cancer target candidate. Expert Opin Ther Targets 14: 935-949, 2010.

3. Maltseva I, Chan M, Kalus I, Dierks T and Rosen SD: The SULFs, extracellular sulfatases for heparan sulfate, promote the migration of corneal epithelial cells during wound repair. PLoS One 8: e69642, 2013.

4. Lamanna WC, Baldwin RJ, Padva M, Kalus I, Ten Dam G, van Kuppevelt TH, Gallagher JT, von Figura K, Dierks T and Merry CL: Heparan sulfate 6- $O$-endosulfatases: Discrete in vivo activities and functional co-operativity. Biochem J 400: 63-73, 2006.

5. Uchimura K, Morimoto-Tomita M, Bistrup A, Li J, Lyon M, Gallagher J, Werb Z and Rosen SD: HSulf-2, an extracellular endoglucosamine-6-sulfatase, selectively mobilizes heparinbound growth factors and chemokines: Effects on VEGF, FGF-1, and SDF-1. BMC Biochem 7: 2, 2006.

6. Zhu C, He L, Zhou X, Nie X and Gu Y: Sulfatase 2 promotes breast cancer progression through regulating some tumor-related factors. Oncol Rep 35: 1318-1328, 2016.

7. Hammond E, Khurana A, Shridhar V and Dredge K: The role of heparanase and sulfatases in the modification of heparan sulfate proteoglycans within the tumor microenvironment and opportunities for novel cancer therapeutics. Front Oncol 4: 195, 2014.

8. Al-Gayyar MM, Abbas A and Hamdan AM: Chemopreventive and hepatoprotective roles of adiponectin (SULF2 inhibitor) in hepatocelluar carcinoma. Biol Chem 397: 257-267, 2016.

9. Gill RM, Michael A, Westley L, Kocher HM, Murphy JI and Dhoot GK: SULF1/SULF2 splice variants differentially regulate pancreatic tumour growth progression. Exp Cell Res 324: 157-171, 2014.

10. Khurana A, Beleford D, He X, Chien J and Shridhar V: Role of heparan sulfatases in ovarian and breast cancer. Am J Cancer Res 3: 34-45, 2013.

11. Lui NS, Yang YW, van Zante A, Buchanan P, Jablons DM and Lemjabbar-Alaoui H: SULF2 expression is a potential diagnostic and prognostic marker in lung cancer. PLoS One 11: e0148911, 2016.

12. Fujita K, Takechi E, Sakamoto N, Sumiyoshi N, Izumi S, Miyamoto T, Matsuura S, Tsurugaya T, Akasaka $K$ and Yamamoto T: HpSulf, a heparan sulfate 6-O-endosulfatase, is involved in the regulation of VEGF signaling during sea urchin development. Mech Dev 127: 235-245, 2010.

13. Plate K: From angiogenesis to lymphangiogenesis. Nat Med 7: 151-152, 2001.

14. Karkkainen MJ and Petrova TV: Vascular endothelial growth factor receptors in the regulation of angiogenesis and lymphangiogenesis. Oncogene 19: 5598-5605, 2000.

15. Sleeman JP and Thiele W: Tumor metastasis and the lymphatic vasculature. Int J Cancer 125: 2747-2756, 2009. 
16. Reis-Filho JS and Schmitt FC: Lymphangiogenesis in tumors: What do we know? Microsc Res Tech 60: 171-180, 2003.

17. Tammela T, Petrova TV and Alitalo K: Molecular lymphangiogenesis: New players. Trends Cell Biol 15: 434-441, 2005.

18. Acs G, Paragh G, Rakosy Z, Laronga $C$ and Zhang PJ: The extent of retraction clefts correlates with lymphatic vessel density and VEGF-C expression and predicts nodal metastasis and poor prognosis in early-stage breast carcinoma. Mod Pathol 25: $163-177,2012$

19. Skobe M, Hawighorst T, Jackson DG, Prevo R, Janes L, Velasco P, Riccardi L, Alitalo K, Claffey K and Detmar M: Induction of tumor lymphangiogenesis by VEGF-C promotes breast cancer metastasis. Nat Med 7: 192-198, 2001.

20. Zhu C, Qi X, Chen Y, Sun B, Dai Y and Gu Y: PI3K/Ak and MAPK/ERK1/2 signaling pathways are involved in IGF-1-induced VEGF-C upregulation in breast cancer. J Cancer Res Clin Oncol 137: 1587-1594, 2011.

21. Joy AA, Ghosh M, Fernandes R and Clemons MJ: Systemic treatment approaches in Her2-negative advanced breast cancer-guidance on the guidelines. Curr Oncol 22 (Suppl 1): S29-S42, 2015.

22. Fina E, Callari M, Reduzzi C, D'Aiuto F, Mariani G, Generali D, Pierotti MA, Daidone MG and Cappelletti V: Gene expression profiling of circulating tumor cells in breast cancer. Clin Chem 61: 278-289, 2015.

23. Takei H, Kurosumi M, Yoshida T, Ninomiya J, Hagiwara Y, Kamimura M, Hayashi Y, Tozuka K, Suemasu K, Inoue K, et al: Current trends of sentinel lymph node biopsy for breast cancer - a surgeon's perspective. Breast Cancer 14: 362-370, 2007.
24. Karpanen T, Wirzenius M, Mäkinen T, Veikkola T, Haisma HJ, Achen MG, Stacker SA, Pytowski B, Ylä-Herttuala S and Alitalo K: Lymphangiogenic growth factor responsiveness is modulated by postnatal lymphatic vessel maturation. Am J Pathol 169: 708-718, 2006.

25. Gu Y, Qi X and Guo S: Lymphangiogenesis induced by VEGF-C and VEGF-D promotes metastasis and a poor outcome in breast carcinoma: A retrospective study of 61 cases. Clin Exp Metastasis 25: 717-725, 2008.

26. Cherng JM, Lin CM, Lin CL, Huang SM, Chang HL, Lee CC, Chiang LC and Chang PY: Effects of VEGF121 and/or VEGF165 gene transfection on collateral circulation development. J Formos Med Assoc 99: 603-611, 2000

27. Harris NC, Davydova N, Roufail S, Paquet-Fifield S, Paavonen K, Karnezis T, Zhang YF, Sato T, Rothacker J, Nice EC, et al: The propeptides of VEGF-D determine heparin binding, receptor heterodimerization, and effects on tumor biology. J Biol Chem 288: 8176-8186, 2013.

28. Okamoto K, Oshika Y, Fukushima Y, Ohnishi Y, Tokunaga T, Tomii Y, Kijima H, Yamazaki H, Ueyama Y, Tamaoki N, et al: Xenografts of human solid tumors frequently express cellular-associated isoform of vascular endothelial growth factor (VEGF) 189. Oncol Rep 6: 1201-1204, 1999.

29. Karnezis T, Shayan R, Caesar C, Roufail S, Harris NC, Ardipradja K, Zhang YF, Williams SP, Farnsworth RH, Chai MG, et al: VEGF-D promotes tumor metastasis by regulating prostaglandins produced by the collecting lymphatic endothelium. Cancer Cell 21: 181-195, 2012. 